\title{
Automatic Thresholding for Frame-Repositioning Using External Tracking in PET Brain Imaging
}

\author{
Oline V. Olesen, Sune H. Keller, Merence Sibomana, Rasmus Larsen, Bjarne Roed, and Liselotte Højgaard
}

\begin{abstract}
Motion correction (MC) in positron emission tomography (PET) brain imaging become of higher importance with increasing scanner resolution. Several motion correction methods have been suggested and so far the Polaris Vicra tracking system has been the preferred one for motion registration.

We present an automated algorithm for dividing PET acquisitions into subframes based on the registered head motion to correct for intra-frame motion with the frame repositioning MC method. The method is tested on real patient data (five ${ }^{11} \mathrm{C}$ SB studies and five ${ }^{11} \mathrm{C}$-PIB studies) and compared with an image based registration method (AIR). Quantitative evaluation was done using a correlation measure. The study shows that MC improves the correlation of the PET images and that AIR performed slightly better than the Polaris Vicra. We found significant intra-frame motion of $1-5 \mathrm{~mm}$ in 9 frames but the correlation was not significantly improved using intra-frame MC.
\end{abstract}

\section{INTRODUCTION}

$\mathrm{P}^{\mathrm{s}}$ OSITRON emission tomography (PET) imaging has improved considerably during the last decade now reaching a close to $1 \mathrm{~mm}$ spatial resolution. With increasing scanner resolution patient motion becomes a more serious problem and thus end up counteracting the technological advances of high resolutions PET scanners such as the High Resolution Research Tomograph (HRRT, Siemens) dedicated brain PET scanner [1].

To remove the blurring and distortions from head motion, different approaches to motion correction (MC) using tracking information have been suggested. These include multiple acquisition frames (MAF) involving repositioning reconstructed frames [2], [3], post-reconstruction image-based deconvolution of overall motion [4], incorporation of the overall measured motion within the system matrix of the reconstruction algorithm [5], [6], and motion-correction of individually detected events [7], [8], with the addition of motion-based normalization correction [9] or modification of the system matrix [10], [11]. Combinations of methods where

Manuscript received May 11, 2010

O. V. Olesen is at Siemens Healthcare, DK-2750 Ballerup, Denmark and the Department of Informatics and Mathematical Modelling, Technical University of Denmark, Denmark and Department of Clinical Physiology, Nuclear Medicine \& PET, Rigshospitalet, Copenhagen University Hospital, University of Copenhagen, Denmark (e-mail: ovol@imm.dtu.dk).

S. H. Keller, M. Sibomana, and L. Højgaard are with Department of Clinical Physiology, Nuclear Medicine \& PET, Rigshospitalet, Copenhagen University Hospital, University of Copenhagen, Denmark.

R. Larsen is with the Department of Informatics and Mathematical Modelling, Technical University of Denmark, Denmark.

B. Roed is at Siemens Healthcare, DK-2750 Ballerup, Denmark. sinograms are corrected within each frame, after which the frames are repositioned have also been reported [12]. For these methods an external tracking system is needed and the Polaris Vicra system has so far been preferred in PET brain imaging.

We present an automatic frame division method using the Polaris Vicra for a modified frame repositioning MC scheme in contrast to [3] where the framing was not based on the registered motions. The frame division is based on predefined framing and the recorded motion with added subframing to handle intra-frame motion. The attenuation map generated from a transmission (TX) scan used for attenuation and scatter correction was aligned to each frame and subframe having distinct motion for $\mathrm{MC}$ reconstruction. After reconstruction all frames where aligned to a reference frame and subframes where joined to give the original framing. In [13] a MC quality control protocol is devised and used with Polaris Vicra data from patient ${ }^{11} \mathrm{C}$-raclopride scans, but most validation of MC methods and systems in literature have been done on simulated data or phantom scans. We have chosen to use patient data for our evaluation to see if the performance of our subframing method and Polaris Vicra is good enough for routine use.

\section{METHODS}

\section{A. PET data acquisition}

Data sets from five healthy volunteers recruited for a ${ }^{11} \mathrm{C}$ PIB kinetic neuroreceptor study and five healthy volunteers recruited for a ${ }^{11} \mathrm{C}$-SB kinetic neuroreceptor were included in our investigation. They were selected from sets of 16 scans on the criteria of largest max. motion recorded with Polaris Vicra during the scan. The study was approved by the Ethics Committee of Copenhagen, Denmark and was in accordance with the Helsinki II declaration. Scans were performed on an ECAT High Resolution Research Tomograph (HRRT, Siemens) dedicated brain PET scanner [1] with a resolution down to $1.4 \mathrm{~mm}$ [14]. The scans were reconstructed with the 3D-OSEM PSF algorithm (16 subsets and 10 iterations). The subjects were comfortably placed on our specially designed mattress while their heads were firmly fixed using a vacuum bag and a Velcro band tightened around the head and the head holder.

\section{B. Motion tracking}

An optical 3D motion tracking system, Polaris Vicra (NDI), was used to register head motions during the scans. The 
system uses a rigid tracking tool with three markers, and the system measures the orientation and position of the tool. The tracking tool was fixed to the forehead along the hair line where skin movements are limited. The fixation method uses a standard band-aid with velcro tape from Apodan Nordic as seen in Fig. 1.

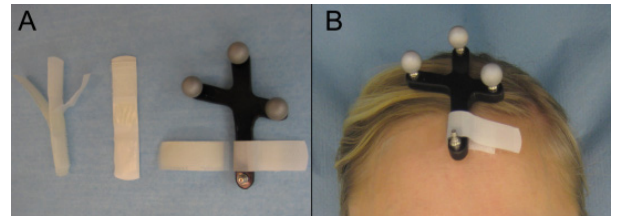

Fig. 1. Fixation of the patient tracking tool.

A position registered by the Polaris must correspond to a known position in the HRRT scanner in order to reposition a given line of response (LOR) or frame correctly. This means that the transformation between the coordinate system of the HRRT image frame and the coordinate system of the Polaris tracker coordinate system has to be established (Fig. 2). We have used a customized reference tool placed inside the back end of the tunnel close to the patient tool.

Using a reference tool, the coordinate alignment refers to the $3 \mathrm{D}$ rigid transformation $\mathbf{A}_{\text {align }}$ that transforms the reference tool into the image frame (transformation between the tracker coordinate system and the image frame).

In [3] and [4] the alignment is obtained using paired emission (EM) scans and Polaris trackings of a point source or a line source placed relative to a tracking tool. We preferred to use TX scans in order to avoid handling radioactive material [15].

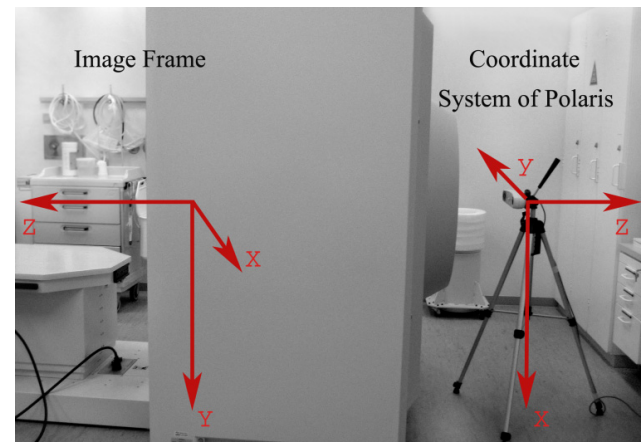

Fig. 2. The Polaris Vicra sensor on a tripod behind the HRRT PET scanner The two coordinate systems are indicated. Note that the image frame by convention is left-handed (we have reversed its y-direction to get two right handed coordinate systems).

\section{Automated frame division}

Dynamic PET studies are generally divided into EM frames based on the expected tracer concentration uptake over time and this predefined framing was kept as basis for the suggested method. The tracking data were divided into subframes of a given time interval $d t$ and tested for inter-frame motion (Fig. 3). If a sub-frame had significant motion larger then $m$ millimeters it was treated as a preserved sub-frame. The EM listmode data were divided into frames and sub- frames based on the original framing and the tracking framing, respectively. After motion correction the sub-frames were joined into the original frames.

The analysis of the tracking was as follows: The tool was chosen as the test point, thus the recorded translations $\left(T_{x}, T_{y}, T_{z}\right)$ were evaluated representing the motion of the brain. This is not the true motion of the complete brain. However, the point in the forehead is highly sensitive to motions, since two primary head motions during scans are a) rotation around the contact point at the back of the head, and b) translation of the head in the axial axis direction. The relative motion of the tool to the reference position $\left(T_{x, \text { ref }}, T_{y, r e f}, T_{z, \text { ref }}\right)$ was estimated as

$$
d\left(T_{x}, T_{y}, T_{z}\right)=\sqrt{\left(T_{x}-T_{x, r e f}\right)^{2}+\left(T_{y}-T_{y, r e f}\right)^{2}+\left(T_{z}-T_{z, r e f}\right)^{2}}
$$

The estimated motion $d\left(T_{x}, T_{y}, T_{z}\right)$ was filtered with a low pass filter with a cut of frequency of $0.5 \mathrm{~Hz}$ to eliminate: noise of the tracking, occasional motion of the tool, and fast oscillations of the tool due to the fixation. Motion from the respiration was not removed with a cut of frequency of 0.5 Hz. Respiratory motion was chosen to be included since it is not necessarily truly periodic around a fixed mean position. It was observed that the respiratory motion was highly varying from person to person and also varied during individual scans.

Then the filtered motion was divided into sub-frames of $d t=10 \mathrm{~s}$ and tested for intra-frame motion. A two-sided $F$ test assuming two independent random samples was performed with the null hypothesis $H_{0}$ that the variances $\sigma_{1}^{2}$ and $\sigma_{2}^{2}$ of the two samples are equal and the alternative hypothesis that the variance of the two samples are different is made. The tested samples are the errors from the mean position during two test-frames.

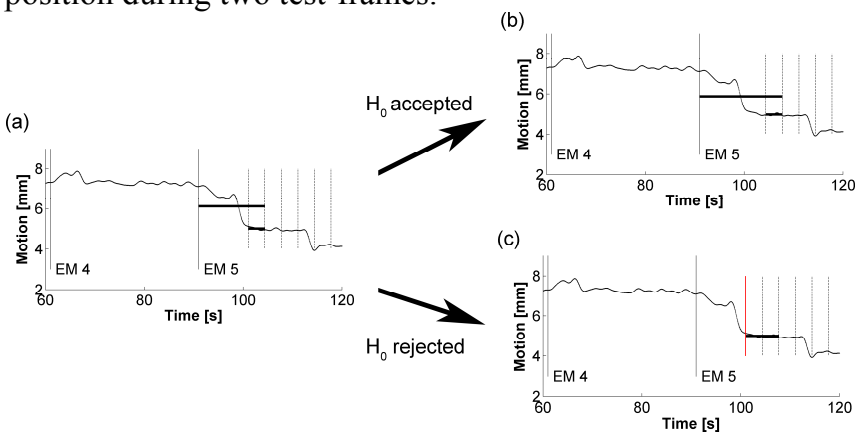

Fig. 3. Principle of the test for intra-frame motion. EM dynamic frames 4 and 5 showing the relative tracked motion of the tool during the test for intraframe motions within frame 5. Vertical black lines: EM frame division. Short vertical black lines: test of subframe division. Red line in (c): preserved subframing due to significant intra-frame motion.

Fig. 3 shows the principle of the test for inter-frame motion where the predefined framing is the long vertical black lines and the sub-framing is the short vertical dashed black lines which are tested. The sub-framing is tested from left to right. Fig. 3(a) on the left hand side shows testing of the first dashed 
line from the left within EM frame no. 5. The horizontal lines represent the mean positions and the lengths of the two testframes (test-frame 1 includes sub-frames 1-4 and test-frame 2 includes only sub-frame 4 . Sub-frames 1 and 2 are not preserved and therefore not visualized). The null hypothesis is rejected if the test size (the ratio between the variances) is:

$$
\frac{\sigma_{1}^{2}}{\sigma_{2}^{2}}<F_{1-\alpha / 2} \quad \text { or } \quad \frac{\sigma_{1}^{2}}{\sigma_{2}^{2}}>F_{\alpha / 2}
$$

where $F_{\alpha}$ is the $F$ distribution with a significance level $\alpha$ of $5 \%$. If $H_{0}$ is rejected the sub-frame is preserved visualized as the red line on Fig. 3(c). Otherwise $H_{0}$ is accepted and the sub-frame is eliminated as shown on the right top Fig. 3(b). In that case the next test-frame is accumulated with the prior one as indicated with the new mean positions and lengths of the test frames (the new horizontal lines).

A second criterion had to be fulfilled in order to preserve a subframe for intra-frame motion. The difference between the mean positions of the subframe itself and the prior frame had to be $>m$. For this study $m$ was set to $1 \mathrm{~mm}$.

The preserved subframes were found with respect to two reference positions: a) we decide if the attenuation map should be aligned to the EM frames prior to reconstruction. Thus the tool position during the first $60 \mathrm{~s}$ of the TX scan is used as a reference position. b) We decide if the reconstructed EM frames should be aligned to the reference EM frame suggested by our algorithm, and thus this EM reference frame is used as a second reference position when estimating the subframing.

For each frame and preserved subframe a quaternion and a translation was representing the transformation for the entire time interval. Since a quaternion is a unit vector a rotation cannot truly be represented by taking means over the quaternions and therefore only one quaternion was extracted (the one closest to the mean position during the time interval). However a post study showed that a mean of $80 \%$ of the quaternions closest to the mean position assuming a unit quaternion was more robust measure. A number of parameters were returned to each frame and used in the motion correction scheme: 1) the root mean square error (RMSE) was defined as the root mean square distances from the tracked position to the mean position of the frame, 2) the size of tracked motion within the TX scan, 3) if the frame had significant motion from the TX scan, 4) the distance to the mean of the reference position, and 5) finally a suggested frame of reference which is described in Section II.F.

\section{Motion corrected reconstruction}

The frames were reconstructed with the frame repositioning motion correction based on the recorded motion during the frames. The detected intra-frame motions were included in the motion correction scheme by the suggested automatic frame division algorithm followed by a reframing where the detected subframes were joined into the original EM framing. The principle of the performed frame repositioning motion correction was: 1) the EM listmode data were histogrammed into frames based on the predefined EM framing and the subframes created due to intra-frame motion; 2) the attenuation map was aligned with the EM frames if the tracked motion during a given EM frame and the TX scan was significantly different and larger than $1 \mathrm{~mm}$ based on the same two-sided $F$-test as for the detection of intra-frame motion; 3 ) the EM frames were reconstructed using the 3D-OSEM PSF algorithm (16 subsets, 10 iterations); 4) the EM frames were aligned to a reference frame; and 5) The subframes with intraframe motion were combined to give the original EM framing.

The attenuation maps and reconstructed EM frames were repositioned based on the external tracking information assuming the patient tool was fixed to the skull and the brain was a rigid body. The complete image transformation referred to as the frame repositioning method is described in [15]. The principle of a frame repositioning is sketched in Fig. 4. The PET image is a grid of points $\left(x_{n}, y_{n}, z_{n}\right)$ expressed in homogeneous coordinates. The complete transformation is a set of transformations where the image is moved into the tracker coordinate system where every coordinate in the rotated image is moved into the corrected grid (reference grid) as illustrated within the dashed box in Fig. 4. Finally the corrected grid is moved back to the image frame $\left(x_{n}^{*}, y_{n}^{*}, z_{n}^{*}\right)$. The complete transformation can be described as

$\left[\begin{array}{llll}x_{n}^{*} & y *{ }_{n} & z^{*}{ }_{n} & 1\end{array}\right]^{\mathrm{T}}=\mathbf{A}_{\text {align }} \mathbf{A}_{\text {ref }} \mathbf{A}_{\text {out }}^{-1} \mathbf{A}_{\text {align }}^{-1}\left[\begin{array}{llll}x_{n} & y_{n} & z_{n} & 1\end{array}\right]^{\mathrm{T}}$

Here $\mathbf{A}_{\text {ref }}$ and $\mathbf{A}_{\text {out }}$ are the recorded transformations of the patient tool at the reference position and a "new" position. $\mathbf{A}_{\text {align }}$ is the alignment transformation between the two coordinate systems. The images were resampled to the discrete corrected grid of coordinates using 3D bilinear interpolation.

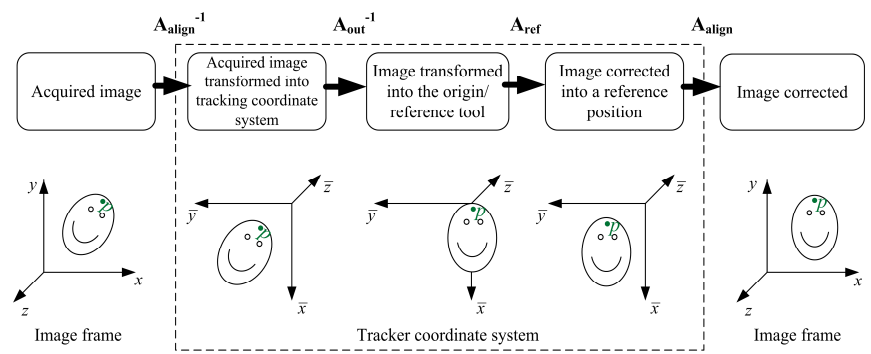

Fig. 4. Sketch of the repositioning of a reconstructed image to a reference position using external tracking information. The scheme involves two coordinate systems and four subtransformations. The point $P$ is the patient tool fixed to the subject.

\section{E. Correlation measure}

We have computed the correlation between the EM reference and each frame in that scan after 3D-OSEM PSF reconstruction with a) the presented method using Polaris Vicra tracking; b) the standard reconstruction without $\mathrm{MC}$; 
and c) image registration based AIR MC method [16] as implemented in the HRRT Users Software. The AIR method cannot register EM images to TX images and thus we used the TX images registered to each reference frame by method a). Alternatively, one can manually correct the TX images as done in [17]. The registration of TX images is of great importance but is also tracer dependant. For Verapamil it is essential for correct scatter correction as shown in [17].

The Polaris Vicra tracking transformations were compared with the estimated transformations using AIR by applying the relative transformations (relative to frame 25) to 1) the point representing the tool position and 2) a point in the backwards in the head $10 \mathrm{~cm}$ behind the tool.

\section{F. Frame of reference}

The method that analyze the tracked head position also suggests a frame of reference found by a score-function with four subfunctions. The function return a score for each EM frame depending on the variables of the subfunctions: 1) duration of the frame $\left.S_{L}(t), 2\right)$ root mean square error (RMSE) of the tracking from a mean position $S_{E}(r m s), 3$ ) time from the injection normalized to the EM scan duration $S_{I}\left(t^{*}\right)$, and 4$)$ the distance to a mean position of the complete EM scan $S_{P}(p)$. Fig. 5 shows the four subfunctions and the score-function was defined as

$$
S(\text { frame })=S_{L}(t)\left(S_{E}(r m s) \cdot w_{E}+S_{I}\left(t^{*}\right) \cdot w_{I}+S_{P}(p) \cdot w_{P}\right)
$$

where $w_{E}, w_{I}$, and $w_{P}$ were weights that could be adjusted as wanted. The returned suggested frame of reference was the frame with the highest score and no subframes if possible. In the ten scans tested, the reference frames selected are frames number 21-32.
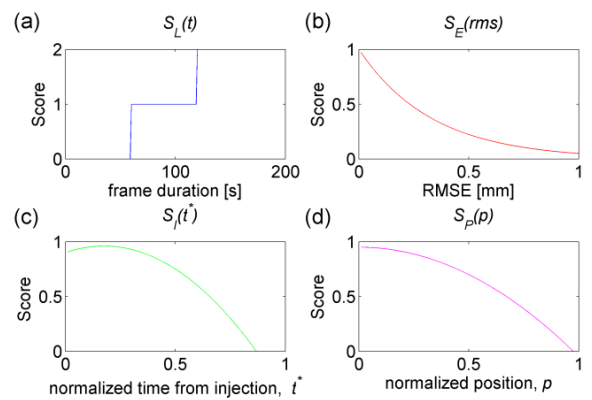

Fig. 5. Subfunctions of the score-function $S$ (frame) . The four plots show the score as a function of: (a) frame duration, (b) RMSE of the tracking, (c) time from injection, and (d) distance to the reference position.

\section{RESULTS}

The five PIB scans have 34 frames $(4 \times 15 \mathrm{~s}, 8 \times 30 \mathrm{~s}, 9 \times 60 \mathrm{~s}$, $2 \times 180 \mathrm{~s}, 8 \times 300 \mathrm{~s}, 3 \times 600 \mathrm{~s})$ and the five SB scans have 38 frames $(6 \times 5 \mathrm{~s}, 10 \times 15 \mathrm{~s}, 4 \times 30 \mathrm{~s}, 5 \times 120 \mathrm{~s}, 5 \times 300 \mathrm{~s}, 8 \times 600 \mathrm{~s})$. One of the PIB scan Polaris Vicra tracks with subframes is shown in Fig. 6 (110 min from the TX scan to the end of the EM scan. The relative motion of the tool is seen as a function of the time with indication of: the predefined framing (black lines), the preserved subframes (red lines), and the mean position of the frames (green/pink lines). Fig. 6(a) shows the motion relative to the first $60 \mathrm{~s}$ of the TX scan end Fig. 6(b) shows the motion relative to the suggested reference frame in this case EM frame number 25. The 'green' frames are significant different from the reference frame and the mean positions are more than $1 \mathrm{~mm}$ from the mean of the reference frame.
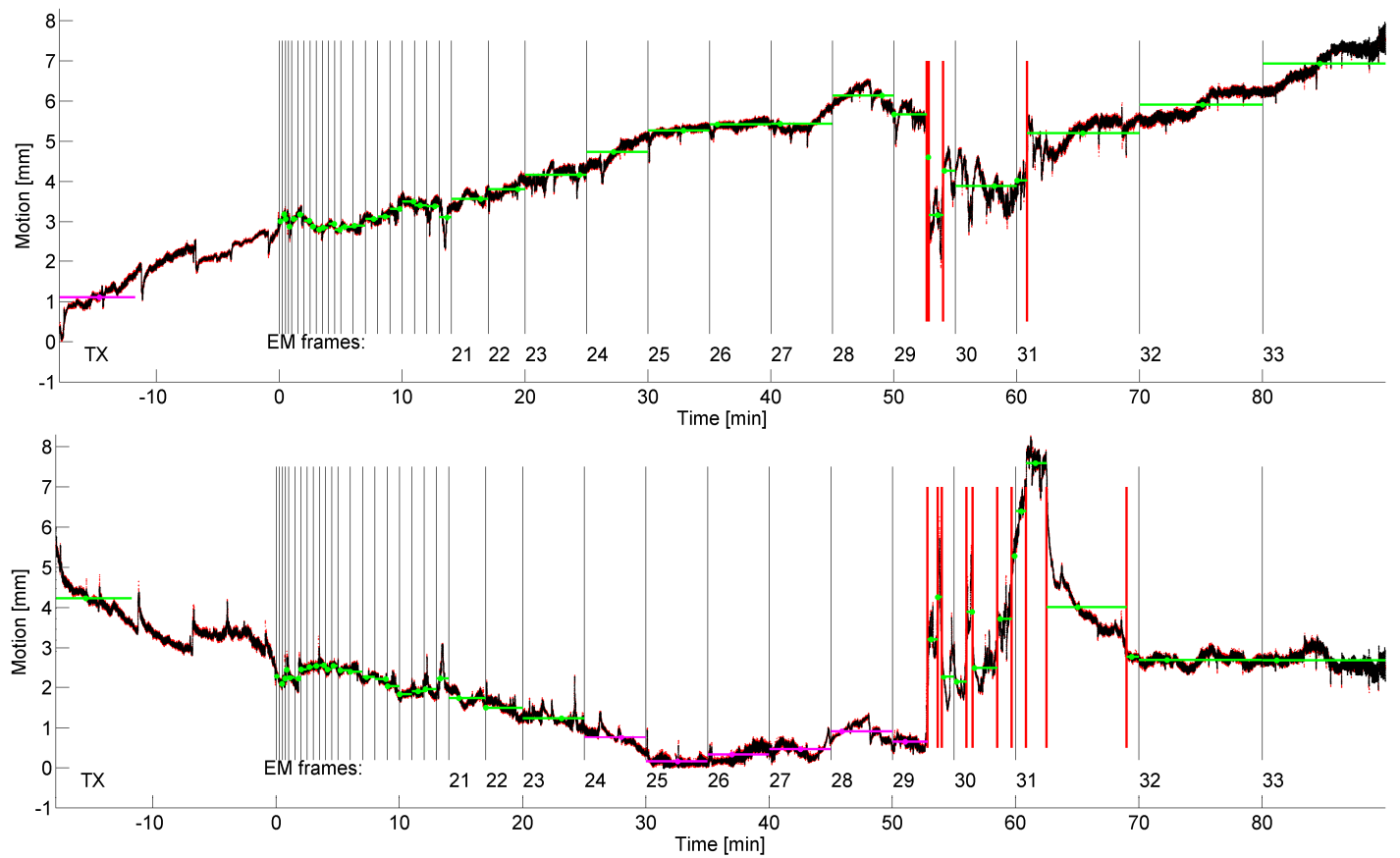

Fig. 6. Tracked motion of the patient tool for one subject (SH) relative to each of the two reference positions. (a) TX scan and (b) the EM reference frame (\#25). Red lines show subframing where both sets, (a) and (b) are joined to the final set of subframes. Green frames have mean motions $>1 \mathrm{~mm}$ relative to the reference position and pink frames have mean motions $<1 \mathrm{~mm}$. 

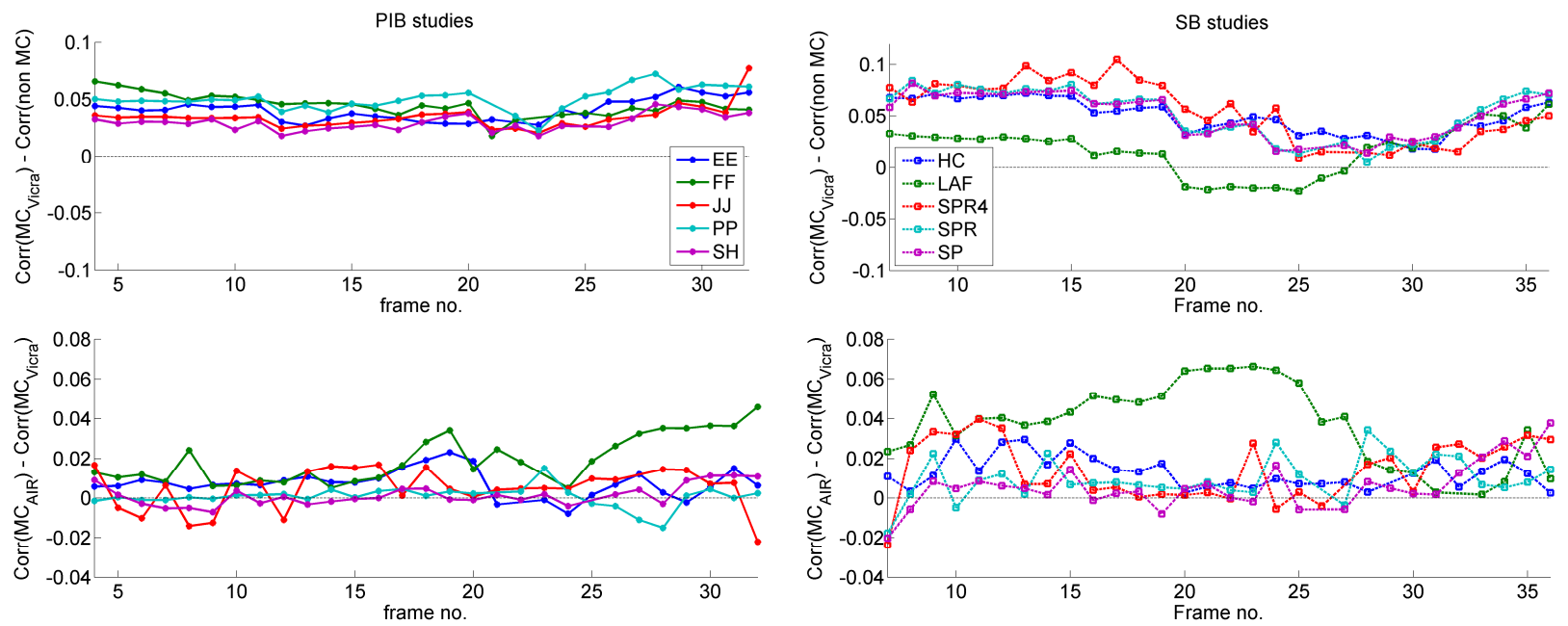

Fig. 7. Correlation results of the five PIB studies (left) and the five SB studies (right). Top: difference of the correlation with and without MC using the Polaris Vicra. Bottom: difference of the correlation with $\mathrm{MC}$ using Polaris Vicra and AIR [Corr( $\left.\left.\mathrm{MC}_{\mathrm{AIR}}\right)-\operatorname{Corr}\left(\mathrm{MC}_{\mathrm{Vicra}}\right)\right]$.

Table I lists the tracked motion for each scan at the position of the tool. The registered motion during each of the ten TX scan was from $0.8 \mathrm{~mm}$ to $5.1 \mathrm{~mm}$ (column 2). The table also lists the maximum motion of the frame mean positions relative to the TX position (column 3) and the EM reference position (column 5). The percentage of frames where the motion are $>3 \mathrm{~mm}$ is given in column 4 (TX) and column 6 (EM). The recorded motions relative to the TX scans $(3.6-10.3 \mathrm{~mm})$ are higher than the recorded motions relative to the EM reference (2.8-8.1 mm). For two subjects PP and LAF the motion of the tool are lower than $3 \mathrm{~mm}$ for all the frames relative to the EM reference position and for all subjects no more than $38 \%$ of the frames have moved more than $3 \mathrm{~mm}$.

Two scans SPR and SPR4 (rescan of the same subject) had noisy motion patterns with many sporadic high peaks and thus the motion threshold for intra-frame motion was increased to $3 \mathrm{~mm}$ for these two scans. Intra-frame motions significant for both of the reference positions were detected in only 9 frames (or 41 frames if significant for only one of the reference positions) of the $360(34 \times 5+38 \times 5)$ frames in total with up to seven subframes in a single original frame. The size of the intra-frame motion was $1-5 \mathrm{~mm}$ with overall corrected motions less than $11 \mathrm{~mm}$. Five subjects had intra-frame motions with $1 \mathrm{~mm}$ motion threshold (3 mm motion threshold for SPR and SRP4). The correlation of the frames with detected intra-frame motion was not significantly improved compared to Polaris Vicra MC without intra-frame motion included.

Fig. 7 shows the results of the correlation for the PIB and SB studies to the left and right respectively. The difference of correlations with and without MC is shown in the top as a function of the frame number. All the curves except for LAF (green) are above zero indicating that the correlation between the reference frame and the actual frame have been improved applying the MC. The plots in the bottom show the difference of the correlation when using Polaris Vicra tracking information and using the AIR transformations [Corr $\left(\mathrm{MC}_{\mathrm{AIR}}\right)$ $\left.\operatorname{Corr}\left(\mathrm{MC}_{\mathrm{Vicra}}\right)\right]$. The curves are close to zero (except for $\mathrm{LAF}$ ) but mostly above zero meaning that the correlation is higher using AIR MC. Table I last column lists the percentage of frames where AIR has a higher correlation.

Fig. 8 shows that the difference between the Polaris Vicra and AIR tracking are relative high for the five SB in the last frames where the frame durations are $5 \mathrm{~min}$ (frames 25-29) and $10 \mathrm{~min}$ (frames 30-37). The curve for LAF has a different path for the two compared positions. The difference at the tool position are below $1 \mathrm{~mm}$ at the position of the tool (Fig. 8a) while the difference increases to $2-7 \mathrm{~mm}$ after frame 27 when looking at a point in the back head (Fig. 8b) indicating that the tool has been detaching partially from the head. For the outlier LAF, videos made from the frames of each reconstruction clearly show a large residual rotational motion in the Polaris Vicra MC which is not seen in the AIR MC video. This supports the tool is detaching from the head. The problem of the tool moving differently from the head is well-known with the Polaris Vicra system [18].

\section{TABLE I. TRACKED MOTION OF THE TOOL POSITION}

\begin{tabular}{|c|c|c|c|c|c|c|}
\hline $\begin{array}{l}\text { Sub- } \\
\text { ject }\end{array}$ & $\begin{array}{c}\text { Motion } \\
\text { during TX }\end{array}$ & $\begin{array}{r}\text { Mo } \\
\text { Max }\end{array}$ & $\begin{array}{l}\text { on to TX } \\
\text { Fr. }>3 \mathrm{~mm}\end{array}$ & $\begin{array}{l}\text { Motic } \\
\text { Max }\end{array}$ & $\begin{array}{l}\text { to EM ref } \\
\text { Fr. }>3 \mathrm{~mm}\end{array}$ & $\begin{array}{c}\text { AIR vs. } \\
\text { Vicra (\%) }\end{array}$ \\
\hline $\mathrm{EE}$ & 5.1 & 10.3 & $79 \%$ & 8.1 & $35 \%$ & $86 \%$ \\
\hline $\mathrm{FF}$ & 2.6 & 8.7 & $65 \%$ & 6.0 & $38 \%$ & $100 \%$ \\
\hline $\mathrm{JJ}$ & 3.2 & 4.5 & $21 \%$ & 4.6 & $21 \%$ & $79 \%$ \\
\hline PP & 4.3 & 6.7 & $62 \%$ & 2.8 & $0 \%$ & $64 \%$ \\
\hline $\mathrm{SH}$ & 1.7 & 5.8 & $29 \%$ & 3.6 & $3 \%$ & $50 \%$ \\
\hline $\mathrm{HC}$ & 1.5 & 4.9 & $21 \%$ & 4.4 & $13 \%$ & $100 \%$ \\
\hline LAF & 1.1 & 4.4 & $29 \%$ & 3.0 & $0 \%$ & $100 \%$ \\
\hline SPR4 & 0.8 & 8.9 & $68 \%$ & 5.5 & $18 \%$ & $86 \%$ \\
\hline SPR & 3.6 & 6.3 & $32 \%$ & 4.5 & $21 \%$ & $90 \%$ \\
\hline SP & 3.3 & 3.6 & $16 \%$ & 3.8 & $15 \%$ & $76 \%$ \\
\hline
\end{tabular}

Registered motions of the five PIB subjects (top) and the five SB subjects (bottom) at the point of the tool position. Column 2: max motion during the TX scan. Column 3-4: mean motion of the EM frames relative to the TX position given by max motion and percentage of frames where the tool have moved $>3 \mathrm{~mm}$. Column 5-6: same as column 3-4 but now the motions are relative the EM reference. The last column lists the percentage of frames where the correlation of $\mathrm{MC}$ with AIR was higher than the correlation of $\mathrm{MC}$ with Polaris Vicra. 


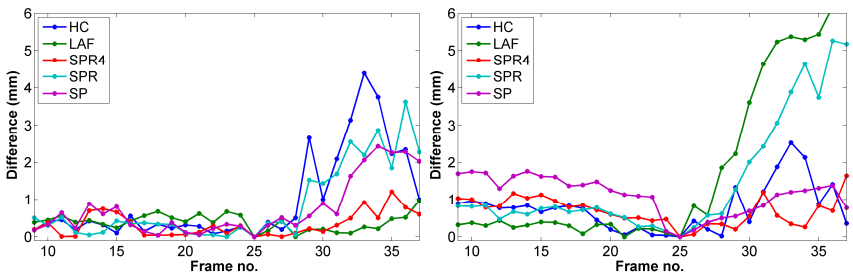

Fig. 8. Difference of the Polaris Vicra tracking and the AIR tracking for the SB subjects. The difference $(\mathrm{mm})$ of a tracked point relative to frame 25 as a function of the frames. Left: the tool position. Right: a point $10 \mathrm{~cm}$ below the tool.

\section{Discussion}

We have presented an automatic method for analyzing and incorporating motion information in the PET reconstruction based on simple frame/subframe repositioning. The method is adaptive for clinical routine. We clearly see an effect of motion compensation on our clinical data: In frames that are not realigned, $\mathrm{MC}$ and non-MC frames have the same correlation as expected, but for corrected frames we see higher correlation to the reference frame for our Polaris Vicra based MC method as well as for AIR MC. For one subject LAF, only AIR MC improves the correlation as the patient tool moves during the scan.

The effect of using our subframing scheme is very little over just using Polaris Vicra with the given framing, and for the majority of frames, AIR MC gives higher correlation (column 7 in Table I). Similar performances of Polaris Vicra MC and AIR MC are reported in [13]. We had expected to see an improvement of correcting for intra-frame motion as simulated in our phantom study based on ${ }^{18}$ F-FDG [15]. However the Polaris Vicra tracking is less accurate on human studies due to the tool fixation and our subjects have limited intra-frame motions with $5 \mathrm{~mm}$ as the most extreme (Fig. 6 bottom). Some plausible reasons why subframing does not improve correlation and why AIR is not surpassed by Polaris Vicra tracking are:

- Due to the fixation problems the patient tool might have moved for other subjects than LAF just to a lesser degree. Fig. 8 shows that the difference in performance between AIR and Polaris gets bigger away from the tool. It might be AIR performing worse, but is more likely tool motion or simply less fidelity of the Polaris transformer away from the tool (due to non-rigidity of the fixation).

- Our patients are very well-bedded and move very little during scans: We selected the $5+5$ out of $16+16$ scans with highest max motion during the scan, the remaining $11+11$ scans having motion peaks of at most 2-4 mm. On more than 100 Polaris tracks studied, we found only two having more than $10 \mathrm{~mm}$ peak motion (EE in the presented dataset being one of them).

- It is non-trivial to select a transformer that represents the motion of a full (sub)frame. This should be obvious looking at e.g. frame 24 or 28 in Fig. 6.

- Correlation might not be a good measure of MC performance, and it might favor AIR that uses a correlation-like measure in it image registration (the quality control developed in [13] shows Polaris being more on a level with AIR than in our study).

- Adding several shorter subframes with low statistics and poorer image quality might cancel out the effect of correcting for motion during the frame.

- Promising results from phantom or simulation studies might not always transfer well to the complexity of human scans.

Besides improving visual image quality for the physicians reading the images, our other main goal of doing $\mathrm{MC}$ is to eliminate any motion induced artifacts in the kinetic analysis of the scans. Thus we analyzed time activity curves (TACs) for 50 regions in the brain in the ten subjects. The regions were obtained using the framework presented in [19]. We did not see any significant differences in the TACs between no MC, Polaris MC and AIR MC.

The two most obvious advantages of using an external tracking system are that you can easily register the TX image with the EM reference frame, and you can detect for intraframe motions, which is required for listmode and sinogram based motion correction.

Before we can do reliable sinogram (or listmode) correction of data, we need an external tracking system that has no tool fixation problems and runs reliably: In $10-20 \%$ of our scans we get no Polaris Vicra tracking, either because the patient tool cannot be registered by the sensor or the tracked data turns out useless for all or parts the scan duration.

The fixation of the tracking tool will always be a major source of error using the Polaris system as it is crucial; a minor rotation of the tool not related to the head has a highly destructive effect on the PET image increasing with the distance to the tool position. Our fixation method is unfortunately no exception.

Having been unable to show significant improvements in TACs with MAF MC (using Polaris Vicra external tracking or AIR) on human patient scans, we are working on a new tracking system without tools. This will hopefully enable us to do sinogram (or LOR) based MC on routine patient studies and improve image quality and kinetic analysis results.

\section{CONCLUSION}

We have presented an automatic frame division method based on external tracking to correct for intra-frame motion with the frame repositioning MC method. The suggested method using the Polaris Vicra was compared with AIR image registration. The methods were evaluated on patients studies based on a quantitative correlation measure. The correlation measure was improved for both of the MC methods. Using AIR resulted in slightly better correlation than using Polaris Vicra. We found that for few cases AIR was much better because the motion registered by Vicra was incorrect due to the tool being insufficiently fixed to the patient and moving 
independently. We believe the general non-rigidity of the tool fixation to patients is a crucial problem using the Polaris system. That in combination us having only $1-5 \mathrm{~mm}$ of intraframe motion are the two major reasons why we did not see significantly improved correlations with the suggested intraframe MC method.

\section{REFERENCES}

[1] K. Wienhard and M. Schmand, "The ECAT HRRT: performance and first clinical application of the new high resolution research tomograph," IEEE Trans. Nucl. Sci., vol. 49, no. 1, p. 104, Feb. 2002.

[2] Y. Picard and C. J. Thompson, "Motion correction of PET images using multiple acquisition frames," IEEE Trans. Med. Imag., vol. 16, no. 2, pp 137-144, 1997.

[3] H. Herzog, L. Tellmann, R. Fulton, I. Stangier, E. R. Kops, K. Bente, C. Boy, R. Hurlemann, and U. Pietrzyk, "Motion Artifact Reduction on Parametric PET Images of Neuroreceptor Binding," J Nucl. Med., vol. 46, no. 6, pp. 1059-1065, Feb. 2005

[4] N. Raghunath, T. L. Faber, S. Suryanarayanan, and J. R. Votaw, "Motion correction of PET brain images through deconvolution: II Practical implementation and algorithm optimization," Phys. Med. Biol., vol. 54, no. 3, pp. 813-829, Feb. 2009.

[5] A. Rahmim, J. C. Cheng, K. Dinelle, M. Shilov, W. P. Segars, O. G. Rousset et al., "System matrix modelling of externally tracked motion," Nucl. Med. Commun., vol. 29, no. 6, pp. 574-581, June 2008.

[6] M. Reyes, G. Malandain, P. M. Koulibaly, M. A. Gonzalez-Ballester, and J. Darcourt, "Model-based respiratory motion compensation for emission tomography image reconstruction," Phys. Med. Biol., vol. 52, no. 12 , pp. 3579-3600, Jul. 2007.

[7] M. Menke and M. S. Atkins, "Compensation methods for head motion detected during PET imaging," IEEE Trans. Nucl. Sci., vol. 43, no. 1, pp. 310-317, 1996.

[8] S.-K. Woo, H. Watabe, Y. Choi, K. M. Kim, C. C. Park, and P. M. Bloomfield, "Sinogram-based motion correction of PET images using optical motion tracking system and list-mode data acquisition," IEEE Trans. Nucl. Sci., vol. 51, no. 3, pp. 782-788, June 2004.

[9] P. Bühler, U. Just, E. Will, and J. Kotzerke, "An accurate method for correction of head movement in PET," IEEE Trans. Med. Imag., vol. 23, pp. 1176-1185, Sep. 2004

[10] R. E. Carson, W. C. Barker, and J. S. Liow, "Design of a motioncompensation OSEM list-mode algorithm for resolution-recovery reconstruction for the HRRT," Conference Record of the IEEE Nuclear Science Symposium and Medical Imaging Conference, vol. 5, pp. 3281$3285,2003$.

[11] A. Rahmim, K. Dinelle, J. C. Cheng, M. A. Shilov, W. P. Segars, S. C. Lidstone et al., "Accurate event-driven motion compensation in highresolution PET incorporating scattered and random events," IEEE Trans. Med. Imag., vol. 27, no. 8, pp. 1018-1033, Aug. 2008.

[12] A. J. Montgomery, K. Thielemans, M. A. Mehta, F. Turkheimer, and S. Mustafovic, "Correction of head movement on PET studies: comparison of methods," J. Nucl. Med., vol. 47, no. 12, pp. 1936-1944, 2006.

13] H. Ngo, K. Dinelle, S. Blinder, N. Vafai, G. Topping, and V. Sossi, "Quality Control Protocol for Frame-to-Frame PET Motion Correction," IEEE Nuclear Science Symposium Conference Record (MIC), IEEE, 2009

[14] O. V. Olesen, M. Sibomana, S. H. Keller, C. Svarer, J. A. Jensen, S. Holm, F. Andersen, and L. Højgaard, "Spatial resolution of the HRRT PET scanner using 3D-OSEM PSF reconstruction," Conference Record of the IEEE Nuclear Science Symposium and Medical Imaging Conference, no. M13-225, 2009.

[15] O. V. Olesen, C. Svarer, M. Sibomana, S. H. Keller, S. Holm, J. A Jensen, F. Andersen, and L. Højgaard, " A moveable phantom design for quantitative evaluation of motion correction studies in high resolution PET scanners," IEEE Trans. Nucl. Sci., vol. 3, no. 57, pp $1116-1124,2010$

[16] R. P. Woods, S. R. Cherry, and J. C. Mazziotta, "Rapid automated algorithm for aligning and reslicing PET images," Journal of Computer Assisted Tomography, 16, pp. 620-633, 1992.
[17] M. C. Huisman et al., " Quantitative Accuracy of the HRRT in an Interscanner Study with the $\mathrm{HR}+$ and $(\mathrm{R})-\left[{ }^{11} \mathrm{C}\right]$ verapamil," IEEE Nuclear Science Symposium Conference Record (MIC), IEEE, 2010.

[18] H. Herzog, L. Tellman, R. Fulton, and U. Pietrzyk, "Motion correction in PET brain studies," IEEE Proc. The Fourth International Workshop on Multidimensional Systems, pp. 178-181, 2005.

[19] C. Svarer, K. Madsen, S. G. Hasselbalch, L. H. Pinborg, S. Haugbol, V.G. Frokjaer, S. Holm, O. B. Paulson, and G. M. Knudsen, "MR-based automatic delineation of volumes of interest in human brain PET images using probability maps," Neuroimage 24 (4):969-79, 2005. 